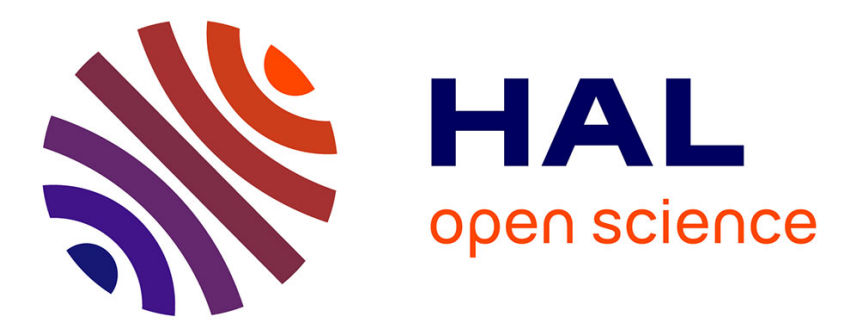

\title{
Energy dissipating structures produced by walls in two-dimensional flows at vanishing viscosity
}

Romain Nguyen van Yen, Marie Farge, Kai Schneider

\section{To cite this version:}

Romain Nguyen van Yen, Marie Farge, Kai Schneider. Energy dissipating structures produced by walls in two-dimensional flows at vanishing viscosity. Physical Review Letters, 2011, 106, pp.184502. 10.1103/PhysRevLett.106.184502 . hal-01022604

\section{HAL Id: hal-01022604 https://hal.science/hal-01022604}

Submitted on 13 Jan 2022

HAL is a multi-disciplinary open access archive for the deposit and dissemination of scientific research documents, whether they are published or not. The documents may come from teaching and research institutions in France or abroad, or from public or private research centers.
L'archive ouverte pluridisciplinaire HAL, est destinée au dépôt et à la diffusion de documents scientifiques de niveau recherche, publiés ou non, émanant des établissements d'enseignement et de recherche français ou étrangers, des laboratoires publics ou privés. 


\title{
Energy Dissipating Structures Produced by Walls in Two-Dimensional Flows at Vanishing Viscosity
}

\author{
Romain Nguyen van yen and Marie Farge \\ LMD-CNRS-IPSL, École Normale Supérieure, Paris, France
}

Kai Schneider

M2P2-CNRS and CMI, Université d'Aix-Marseille, Marseille, France

(Received 13 October 2010; published 3 May 2011)

\begin{abstract}
We perform numerical experiments of a dipole crashing into a wall, a generic event in two-dimensional incompressible flows with solid boundaries. The Reynolds number ( $\mathrm{Re}$ ) is varied from 985 to 7880 , and no-slip boundary conditions are approximated by Navier boundary conditions with a slip length proportional to $\mathrm{Re}^{-1}$. Energy dissipation is shown to first set up within a vorticity sheet of thickness proportional to $\mathrm{Re}^{-1}$ in the neighborhood of the wall, and to continue as this sheet rolls up into a spiral and detaches from the wall. The energy dissipation rate integrated over these regions appears to converge towards Re-independent values, indicating the existence of energy dissipating structures that persist in the vanishing viscosity limit.
\end{abstract}

PACS numbers: $47.27 . \mathrm{N}-$

Flows whose energy dissipation rate is almost independent of small microscopic coupling coefficients are of central interest in fluid dynamics. For example, they are observed with Landau damping in collisionless plasmas, with reconnection in magnetized fluids or plasmas, and with fully developed turbulence in incompressible neutral fluids. Despite its ubiquity, the phenomenon is still often called "anomalous dissipation." One way of approaching it has been to look for generic structures whose existence in the flow is sufficient to imply nonzero energy dissipation. Shocks in compressible flows are a relatively well understood example of such energy dissipating structures, but their well-established theory [1] is an exception.

In this Letter, we focus on two-dimensional incompressible flows in contact with solid boundaries, for which the understanding is, comparatively, at an embryonic stage. Working with the inviscid potential flow equations, later generalized as the Euler equations, d'Alembert in 1752 came up with the famous paradox bearing his name [2] that the flow would exert no drag force onto solid obstacles. The Navier-Stokes equations (NSE) were then derived during the 19th century by including friction effects. It was gradually realized that the paradox came from the singular nature of the vanishing viscosity limit, mostly due to the no-slip boundary condition imposed along the solid boundary. In 1904, Prandtl [3] resolved the paradox in the very special case of flows in which the effects of viscosity are confined to a boundary layer of thickness proportional to $\mathrm{Re}^{-1 / 2}$ in the neighborhood of the wall, where Re is the Reynolds number. He was able to compute a drag coefficient, and hence also an energy dissipation rate, which are both proportional to $\mathrm{Re}^{-1 / 2}$ in the vanishing viscosity limit. Prandtl's theory does not apply when the boundary layer detaches from the wall, because the Euler equations can then no longer be used to describe the flow, even far from the solid boundary. In 1984 Kato [4] proved mathematically that, in the vanishing viscosity limit, the energy dissipation rate tends to zero if and only if the solution of the NSE converges to the solution of the Euler equations with the same initial data. He also proved in the same paper that, for dissipation to occur anywhere in the flow at any time, at least some dissipation had to occur within a very thin boundary layer of thickness proportional to $\mathrm{Re}^{-1}$ in the neighborhood of the wall.

The generic event that we focus on in this Letter consists in the collision of a vorticity dipole into a solid wall, a classical object of experimental studies (see, e.g., [5]), which has also been used for benchmarking several numerical methods [6-9]. A first specific study of energy dissipation appeared in [10], where the NSE with no-slip boundary conditions were approximated using Galerkin discretization over a basis of Chebychev polynomials, both in the wall-parallel and wall-normal directions. The numerical solutions satisfied the no-slip boundary conditions to machine accuracy, but it was later found out [7] that they were not converged beyond the collision time.

In contrast, to cope with Kato's theorem, we have focused on using a numerical scheme which resolves scales at least as fine as $\mathrm{Re}^{-1}$. This stringent requirement on resolution has not been enforced, to our knowledge, in any previous numerical experiments at similar Reynolds numbers. The best way that we have found to comply with it was to work with a numerical model known as volume penalization $[11,12]$. The counterpart, as we shall show in detail below, is that the no-slip boundary conditions are replaced by Navier boundary conditions with a slip length tending to zero when $\operatorname{Re} \rightarrow \infty$. 
The initial value problem for the NSE with volume penalization (PNSE) can be written as follows [11]:

$$
\begin{aligned}
& \partial_{t} \mathbf{u}+(\mathbf{u} \cdot \boldsymbol{\nabla}) \mathbf{u}=-\boldsymbol{\nabla} p+\nu \boldsymbol{\nabla}^{2} \mathbf{u}-\frac{1}{\eta} \chi \mathbf{u}, \\
& \boldsymbol{\nabla} \cdot \mathbf{u}=0, \quad \mathbf{u}(\cdot, t=0)=\mathbf{u}_{0},
\end{aligned}
$$

where $\mathbf{u}(\mathbf{x}, t)$ and $p(\mathbf{x}, t)$ are, respectively, the velocity and pressure fields defined for $\mathbf{x}$ in the unit torus $\mathbb{T}^{2}=(\mathbb{R} / \mathbb{Z})^{2}$ and $t \geq 0, \nu$ is the kinematic viscosity, $\chi$ is a mask function which equals 0 in the fluid part $\Omega$ of $\mathbb{T}^{2}$ and 1 elsewhere, and $\eta$ is the penalization parameter which needs to be sufficiently small in order to impose that the velocity almost vanishes outside $\Omega$. In the following we shall take $\Omega=] 0.05,0.95[\times \mathbb{T}$, thus modeling a periodic channel. To be consistent with previous studies $[6,8]$, the Reynolds number is defined as $\operatorname{Re}=\frac{U L}{\nu}$, where $L$ is the half-width of the channel ( $L=0.45$ in our units) and $U$ is the initial rms velocity. Note that both the NSE and PNSE are well posed in two dimensions; see [13].

Our main quantity of interest will be the kinetic energy $e(\mathbf{x}, t)=\frac{1}{2}|\mathbf{u}(\mathbf{x}, t)|^{2}$ which evolves pointwise according to

$$
\partial_{t} e+\nabla \cdot((e+p) \mathbf{u})=-\nu|\nabla \mathbf{u}|^{2}-\frac{1}{\eta} \chi \mathbf{u}^{2}+\nu \Delta e,
$$

where the term $\nu|\nabla \mathbf{u}|^{2}$ will be called the local energy dissipation rate, as in [4]. The total energy $E=\int_{\mathbb{T}^{2}} e$, satisfies

$$
\frac{d E}{d t}+2 \nu Z+\frac{1}{\eta} \int_{\mathrm{T}^{2}} \chi \mathbf{u}^{2}=0,
$$

where $Z=\frac{1}{2} \int_{\mathbb{T}^{2}}|\nabla \mathbf{u}|^{2}$ is the enstrophy.

For $\eta \rightarrow \infty$, the PNSE reduces to the NSE in $\mathbb{T}^{2}$, and the solution then satisfies the following scaling for energy dissipation [14], which we call wall-less scaling:

$$
E\left(t_{2}\right)-E\left(t_{1}\right) \propto \mathrm{Re}^{-1} .
$$

For $\eta \rightarrow 0$ and fixed Re, it has been proven mathematically $[15,16]$ that the PNSE approaches the NSE in $\Omega$ with no-slip boundary conditions. This convergence has been checked numerically [8] for the dipole-wall collision at $\operatorname{Re}=1000$.

The initial data that led to a dipole-wall collision were introduced in [6]. It is conveniently defined by its vorticity field $\omega_{0}=\boldsymbol{\nabla} \times \mathbf{u}_{0}$, for which the analytical expression is
TABLE I. Parameters of numerical experiments.

\begin{tabular}{lcccc}
\hline \hline $\operatorname{Re}$ & 985 & 1970 & 3940 & 7880 \\
\hline$N$ & 2048 & 4096 & 8192 & 16384 \\
$\eta$ & $4 \times 10^{-5}$ & $2 \times 10^{-5}$ & $10^{-5}$ & $0.5 \times 10^{-5}$ \\
\hline \hline
\end{tabular}

$$
\omega_{0}(\mathbf{x})=\omega_{e} \sum_{i=0}^{1}(-1)^{i}\left(1-\frac{\left|\mathbf{x}-\mathbf{a}_{i}\right|^{2}}{r^{2}}\right) e^{-\left|\mathbf{x}-\mathbf{a}_{i}\right|^{2} / r^{2}},
$$

where $\mathbf{a}_{0}=(0.445,0.5), \mathbf{a}_{1}=(0.555,0.5), r=0.045$, and $\omega_{e}=299.5$, so that $U=0.443$. Note that in addition to the numerical scheme, two things differ from [10]: the initial distance between the two vortices is slightly larger, and the domain is a channel instead of a square container.

To benefit from optimal efficiency, we work with the vorticity formulation of the PNSE in divergence form [12]. Moreover, in order to enforce the energy balance equation (3) to round-off accuracy, the mask $\chi$ is mollified over about 20 grid points so that its Fourier transform is compactly supported. The equations can then be discretized using a pseudospectral scheme [16] with a finite number $K$ of Fourier modes. To avoid aliasing errors, the grid over which the products are evaluated has $N=3 K$ points in each direction. For time discretization we have used an explicit third order low storage Runge-Kutta scheme, with an integrating factor method ([17], p. 111) to take into account the viscous term. The duration $\delta t$ of each time step is adjusted to ensure stability. The parameters of the four numerical experiments whose results are reported in this Letter are listed in Table I. Our choices of resolution are dictated by the requirement to resolve scales as fine as $\operatorname{Re}^{-1}$, and we take $\eta$ as small as possible but such that it does not constrain $\delta t$ more than the Courant-Friedrichs-Lewy condition does.

Figure 1 depicts the vorticity field in the subdomain $[0.708,0.962] \times[0.5,0.754]$, at $t=0.36,0.4,0.45$, and 0.495 , for $\operatorname{Re}=7880$, computed with $N^{2}=16384^{2}$ grid points. Since the vorticity field is antisymmetric with respect to $x_{2}=0.5$, we need only describe its evolution in the upper half of the domain. We observe that, as the positive vortex of the impinging dipole propagates rightwards, a sheet of negative vorticity is created on the wall. The sheet starts to roll up after having reached an

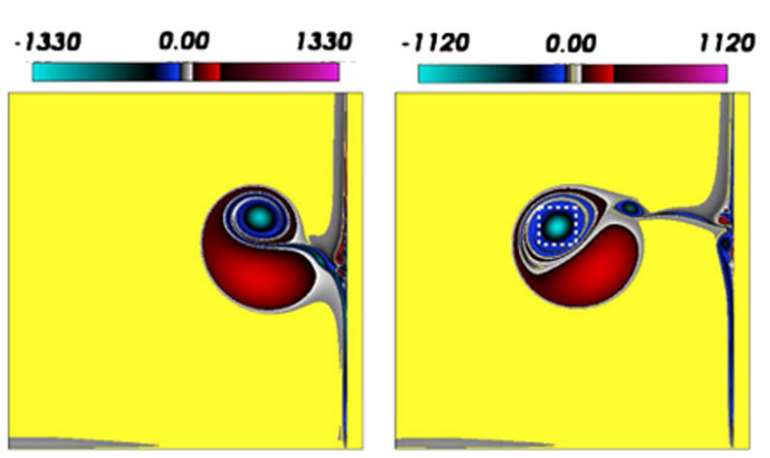

FIG. 1 (color online). Vorticity in the subdomain $[0.708,0.962] \times[0.5,0.754]$ at $t=0.36,0.4,0.45$, and 0.495 (left to right) for $\mathrm{Re}=7880$. The white dotted box at $t=0.495$ frames region $B$ (see text). Black pixels correspond to $\omega= \pm 300$ in all pictures. 

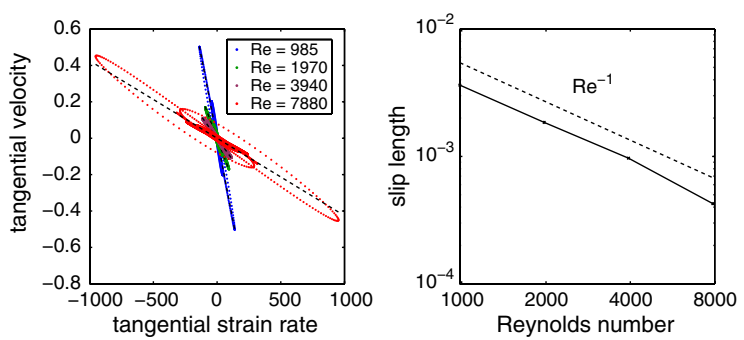

FIG. 2 (color online). Left: Tangential velocity $u_{2}$ versus tangential strain rate $\partial_{1} u_{2}$. The dashed lines are least squares linear fits. Right: Slip length $\alpha$ defined by (6) as a function of Re.

intensity of about 17 times the initial vorticity maximum, and a strong vortex is hence produced. The produced vortex then pairs with the impinging vortex to form a secondary dipole which bounces back from the wall.

Before presenting further results, we would like to dwell on the issue of boundary conditions. We concentrate on the wall facing the impinging dipole and, to avoid grid effects, we define its position as the isoline $\chi=0.02$ along which we interpolate the fields. The value 0.02 is somewhat arbitrary, but its order of magnitude can be justified by balancing the penalization term $\frac{1}{\eta} \chi \mathbf{u}$ with the viscous term $\nu \Delta \mathbf{u}$ in the PNSE. With this definition, the wall-normal velocity $u_{1}$ is smaller than $10^{-3}$ independently of $\mathrm{Re}$, so that to a good accuracy there is no flow through the boundary, as expected. But the wall-parallel velocity $u_{2}$ reaches values of order 0.1 , to be compared to the initial rms velocity $U=0.443$. Plotting $u_{2}$ as a function of $\partial_{1} u_{2}$ for $t=0.495$ along the wall (Fig. 2, left) reveals a relationship of the form

$$
u_{2}+\alpha(\operatorname{Re}, \eta, N) \partial_{1} u_{2} \simeq 0 \text {, }
$$

with a correlation coefficient higher than 0.98 in all cases. Hence, restricted to the domain $\chi<0.02$, u almost satisfies the NSE with Navier boundary conditions and with a slip length $\alpha$. Values of $\alpha$ obtained from least squares fits are shown in Fig. 2 (right) as a function of Re, where we observe that $\alpha$ is approximately proportional to $\mathrm{Re}^{-1}$.
We now come to our main results concerning energy dissipation. In Fig. 3 (left), $E(t=0.2)-E(t=0)$ and $E(0.495)-E(0.39)$ are plotted versus Re. During the time interval $[0,0.2]$, the effect of the boundary is still negligible and the wall-less scaling (4) is recovered. During [0.39, 0.495], we find that the Prandtl scaling $E(0.495)-E(0.39) \propto \mathrm{Re}^{-1 / 2}$ nearly holds. To give more weight to the effects of the boundary, we now compute the enstrophy increase during the same time intervals, i.e., respectively, [0, 0.2] and [0.39, 0.495] (Fig. 3, middle). We observe that $Z(0.495)-Z(0.39) \propto \mathrm{Re}$, suggesting that in that time interval the global energy dissipation rate $2 \mathrm{ZRe}^{-1}$ does not go to zero in the vanishing viscosity limit. This cannot be seen on the curve of $E(0.495)-E(0.39)$ because the effect of the bulk dissipation is still dominant at the Reynolds numbers considered here. However, since $E(0.495)-E(0.39) \approx 2 \nu \int_{0.39}^{0.495} Z(t) d t$, we expect that at higher $\mathrm{Re}$, the dissipative scaling $E(0.495)-E(0.39) \propto \mathrm{Re}^{0}$ holds. To provide more conclusive evidence of this, we isolate two regions where energy is actually dissipated: region $A$, a vertical slab of width $10 N^{-1}$ inside the fluid domain along the isoline $\chi=0.02$, and region $B$, a square box of side length 0.025 around the center of the main structure that has detached from the wall at $t=0.495$ (dotted box in Fig. 1, right). The energy dissipation rate, as defined by (2), is integrated, respectively, over the domain $A$ or $B$ and plotted versus Re in Fig. 3 (right). It can be seen that in both cases the dependence on $\operatorname{Re}$ becomes weak for $\operatorname{Re} \gtrsim 2000$.

The understanding of these results is facilitated by following some Lagrangian particles in the flow. Particles were advected using a bicubic interpolation of the velocity field and third order Runge-Kutta time integration. We now describe the evolution of the energy dissipation rate $\nu|\nabla \mathbf{u}(\mathbf{x}, t)|^{2}$ along three selected trajectories for $\operatorname{Re}=3940$ (Fig. 4). The first striking feature is that it displays a strong maximum for two particles which start from the wall (green and red), occurring when they are still in region $A$, near the wall. In contrast, there is little dissipation along the third trajectory (blue), which starts away from the wall and never enters its vicinity, i.e., region $A$. At later times, energy dissipation goes back to much smaller values for one of the trajectories that approached the wall
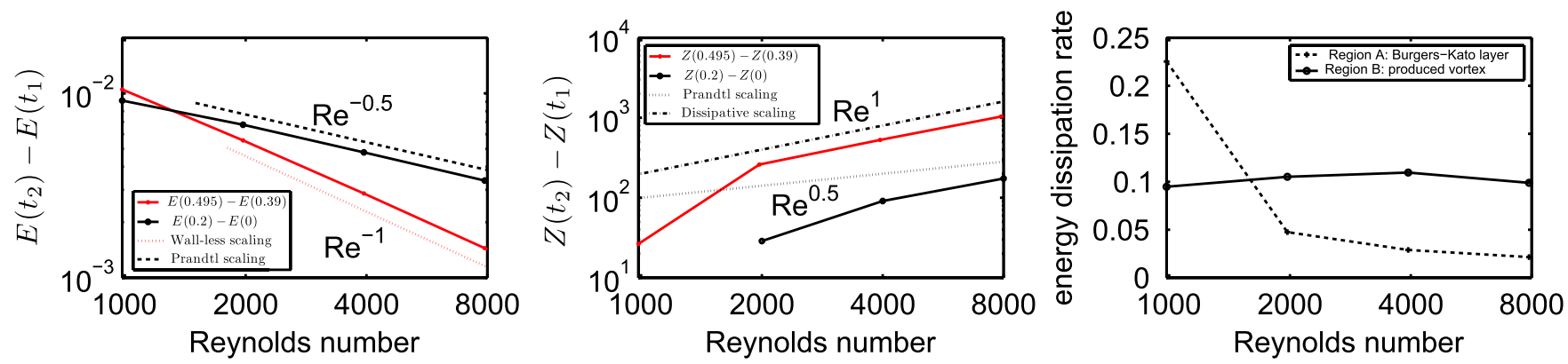

FIG. 3 (color online). Left and middle: Energy dissipation (respectively, enstrophy increase) as a function of Re for $t \in[0,0.2]$ and $t \in[0.39,0.495]$. Right: Instantaneous energy dissipation as a function of $\operatorname{Re}$ at $t=0.495$ in regions $A$ and $B$ (see text). 


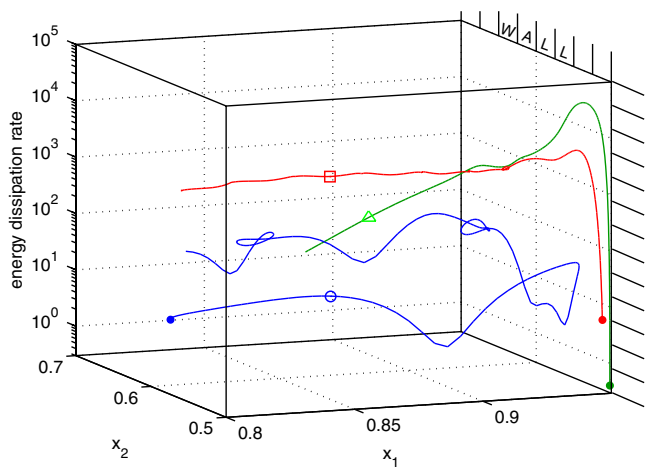

FIG. 4 (color online). Energy dissipation rate $\nu\left|\nabla \mathbf{u}\left(x_{1}(t), x_{2}(t), t\right)\right|^{2}$ versus particle position $\left[x_{1}(t), x_{2}(t)\right]$ for $t \in[0.3,0.495]$ along three Lagrangian trajectories, at $\operatorname{Re}=3940$. The full circles indicate the positions at $t=0.3$. The hollow circle, square, and triangle mark the blue, red, and green trajectories, respectively.

(green), while it is still 1 order of magnitude larger for the other one (red), because the particle is trapped inside the strong vortex produced at the wall (corresponding to region $B$ at $t=0.495$ ).

We have shown that the dipole-wall collision, a wellknown generic event in two-dimensional incompressible flows, is a good candidate for having nonzero energy dissipation in the vanishing viscosity limit. We have imposed Navier boundary conditions with a slip length proportional to $\mathrm{Re}^{-1}$. Note that the convergence of solutions to the NSE under Navier boundary conditions with a slip length varying as $\alpha=\operatorname{Re}^{-\gamma}$ to solutions of the Euler equations has been proven mathematically for $\gamma<0.5$ [18], but that the case $\gamma \geq 0.5$ is still open. Our results suggest that convergence does not hold for $\gamma \approx 1$, due to the same scenario that is implied by Kato's theorem, namely, that energy keeps being dissipated in a very thin boundary layer of thickness proportional to $\mathrm{Re}^{-1}$, orders of magnitude thinner than the Prandtl boundary layer, and which was also predicted by Burgers [19] to explain the observed behavior of the drag force. We propose to call it the Burgers-Kato boundary layer. In addition, we have shown that energy dissipation continues within spiral structures detaching from the boundary, and is likely to occur only along Lagrangian trajectories that "crash" into the wall when $\operatorname{Re} \rightarrow \infty$. We argue further that energy dissipating structures may be common in 2D wall-bounded flows, with slip or no-slip boundary conditions, as long as the slip length is small enough. They could perhaps be observed in soap film experiments or in oceanic flows. These structures are not encompassed by existing statistical theories of 2D turbulent flows [20-22]. Understanding the statistical properties of flows containing energy dissipating structures is thus an open question of great relevance to a theory of $2 \mathrm{D}$ wall-bounded turbulence [23].

We heartily thank Claude Bardos, whose guidance was essential for the completion of this work. We are also grateful to Gregory Eyink, Dmitry Kolomenskiy, Anna Mazzucato, Helena Nussenzveig-Lopes, Qiu Yanqi, Xin Zhouping, and Yin Qizheng for fruitful discussions. We would like to express our gratitude to one of the referees for his or her insightful report. M. F. and R. N. v. y. are thankful to the Wissenschaftskolleg zu Berlin for its hospitality. We thank IDRIS-CNRS for providing computing facilities. This work was carried out within the framework of the European Fusion Development Agreement and the French Research Federation for Fusion Studies. It is supported by the European Communities under the contract of Association between Euratom and CEA.

[1] P. D. Lax, Hyperbolic Systems of Conservation Laws and the Mathematical Theory of Shock Waves (SIAM, Philadelphia, PA, 1973).

[2] J. le Rond d'Alembert, in Essai d'une nouvelle théorie de la résistance des fluides (David l'aîné, Paris, 1752), electronic version available from Gallica, Bibliothèque Nationale de France.

[3] L. Prandtl, in Proceedings of the 3rd International Congress of Mathematicians, Heidelberg, 1904, edited by A. Krazer (Taubner, Leipzig, 1905), p. 484.

[4] T. Kato, in Proceedings of the Seminar on Nonlinear Partial Differential Equations (MSRI, Berkeley, 1984) pp. 85-98.

[5] J. Flor and G. J. van Heijst, J. Fluid Mech. 279, 101 (1994).

[6] P. Orlandi, Phys. Fluids A 2, 1429 (1990).

[7] H. Clercx and C.-H. Bruneau, Comput. Fluids 35, 245 (2006).

[8] G. Keetels, U. D’Ortona, W. Kramer, H. Clercx, K. Schneider, and G. J. van Heijst, J. Comput. Phys. 227, 919 (2007)

[9] W. Kramer, H. Clercx, and G. J. van Heijst, Phys. Fluids 19, 126603 (2007).

[10] H. J. H. Clercx and G. J. F. van Heijst, Phys. Rev. E 65, 066305 (2002).

[11] E. Arquis and J. Calgagirone, C.R. Acad. Sci. Ser. Gen., Ser. 2 299, 1 (1984).

[12] K. Schneider and M. Farge, Phys. Rev. Lett. 95, 244502 (2005).

[13] C. Foiaš, O. Manley, R. Rosa, and R. Temam, NavierStokes Equations and Turbulence (Cambridge University Press, Cambridge, England, 2001).

[14] J. T. Beale and A. Majda, Math. Comput. 37, 243 (1981).

[15] P. Angot, C.-H. Bruneau, and P. Fabrie, Numer. Math. 81, 497 (1999).

[16] G. Carbou and P. Fabrie, Adv. Differ. Equat. 8, 1453 (2003).

[17] L. Trefethen, Spectral Methods in Matlab (SIAM, Philadelphia, PA, 2000).

[18] Z.P. Xin (private communication).

[19] J. M. Burgers, Proc. K. Ned. Akad. Wet. 26582 (1923).

[20] L. Onsager, Nuovo Cimento 6, 279 (1949).

[21] R. H. Kraichnan, Phys. Fluids 10, 1417 (1967).

[22] G. K. Batchelor, Phys. Fluids 12, II-233 (1969).

[23] G. L. Eyink, Physica (Amsterdam) 237D, 1956 (2008). 